\title{
Renormalization approach to many-particle systems
}

\author{
K. W. Becker, A. Hübsch, and T. Sommer \\ Institut für Theoretische Physik, Technische Universität Dresden, D-01062 Dresden, Germany
}

(October 30, 2018)

\begin{abstract}
This paper presents a renormalization approach to many-particle systems. By starting from a bare Hamiltonian $\mathcal{H}=\mathcal{H}_{0}+\mathcal{H}_{1}$ with an unperturbed part $\mathcal{H}_{0}$ and a perturbation $\mathcal{H}_{1}$, we define an effective Hamiltonian which has a band-diagonal shape with respect to the eigenbasis of $\mathcal{H}_{0}$. This means that all transition matrix elements are suppressed which have energy differences larger than a given cutoff $\lambda$ that is smaller than the cutoff $\Lambda$ of the original Hamiltonian. This property resembles a recent flow equation approach on the basis of continuous unitary transformations. For demonstration of the method we discuss an exact solvable model, as well as the Anderson-lattice model where the well-known quasiparticle behavior of heavy fermions is derived.
\end{abstract}

PACS numbers: 71.10.Fd, 71.27.+a, 75.20.Hr, 75.30.Mb

\section{INTRODUCTION}

If one considers a many-particle problem one would like to diagonalize the Hamiltonian so that observables of interest can be calculated. However, only a few models can be solved explicitly so that often one has to use approximations or numerical approaches. Recently Wegner and coworkers [1,2] and Głazek and Wilson [3,4] proposed to solve the eigenvalue problem of a many-body system by transforming the Hamiltonian in such a way that it becomes more and more diagonal. The method is based on the introduction of continuous unitary transformations and is formulated in terms of differential equations for the parameters of the Hamiltonian. These equations are called flow equations.

In this paper we present a renormalization approach which is directly based on perturbation theory for the Hamiltonian instead of using a formulation in terms of differential equations. The starting point is a manyparticle Hamiltonian $\mathcal{H}=\mathcal{H}_{0}+\mathcal{H}_{1}$. It is assumed that the eigenstates and eigenenergies of the unperturbed part $\mathcal{H}_{0}$ are known. The interaction $\mathcal{H}_{1}$ usually prevents the solution of the full eigenvalue problem of $\mathcal{H}$. In Sec. II, we shall derive an effective Hamiltonian $\mathcal{H}_{\lambda}$ on the basis of a perturbational approach. The obtained Hamiltonian $\mathcal{H}_{\lambda}$ has no matrix elements (with respect to the unperturbed Hamiltonian $\mathcal{H}_{0}$ ) which belong to transitions larger than a given energy cutoff $\lambda$. In contrast to standard perturbation theory no vanishing energy denominators appear. In Sec. III, the result for $\mathcal{H}_{\lambda}$ will be used to formulate a $\lambda$-dependent renormalization scheme by repeatedly applying a stepwise transformation on the effective Hamiltonian. In an infinitesimal formulation the renormalization of the Hamiltonian will be described by flow equations. At the end of this section a comparison of our approach with those of Wegner [1] and of Głatzek and Wilson [3,4] is given. For demonstration of the renormalization approach in Sec. IV the exactly solvable FanoAnderson model $[6,7]$ is discussed. This model describes two types of interacting electrons without correlations and the exact result will be obtained in the framework of the renormalization approach. Finally, in Sec. V the application to the Anderson-lattice model [6] is discussed in order to derive the well-established quasiparticle behavior of heavy fermions. The conclusions in Sec. VI conclude the paper.

\section{EFFECTIVE HAMILTONIAN AND PERTURBATION THEORY}

Let us start from a decomposition of a given manyparticle Hamiltonian $\mathcal{H}$ into an unperturbed part $\mathcal{H}_{0}$ and into a perturbation $\mathcal{H}_{1}$

$$
\mathcal{H}=\mathcal{H}_{0}+\varepsilon \mathcal{H}_{1}=: H(\varepsilon) .
$$

We assume that the eigenvalue problem of $\mathcal{H}_{0}$ is known

$$
\mathcal{H}_{0}|n\rangle=E_{n}^{(0)}|n\rangle \text {. }
$$

$\mathcal{H}_{1}$ is the interaction. Its presence usually prevents a solution of the eigenvalue problem of the full Hamiltonian. The parameter $\varepsilon$ accounts for the order of perturbation processes discussed below. Let us define a projection operator $\mathbf{P}_{\lambda}$ by

$$
\mathbf{P}_{\lambda} A=\sum_{\left|E_{n}^{(0)}-E_{m}^{(0)}\right| \leq \lambda}|n\rangle\langle m|\langle n|A| m\rangle .
$$

Here, $\mathbf{P}_{\lambda}$ is a superoperator acting on usual operators $A$ of the unitary space. It projects on that part of $A$ which is formed by all dyads $|n\rangle\langle m|$ with energy differences $\left|E_{n}^{(0)}-E_{m}^{(0)}\right|$ less or equal to a given cutoff $\lambda$, where $\lambda$ is smaller than the cutoff $\Lambda$ of the original model. Note that in Eq. (3) neither $|n\rangle$ nor $|m\rangle$ have to be low-energy eigenstates of $H_{0}$. However, their energy difference has to be restricted to values $\leq \lambda$. Furthermore, it is useful to define the projection operator

$$
\mathbf{Q}_{\lambda}=\mathbf{1}-\mathbf{P}_{\lambda}
$$


which is orthogonal to $\mathbf{P}_{\lambda} \cdot \mathbf{Q}_{\lambda}$ projects on high-energy transitions larger than the cutoff $\lambda$.

The goal of the present method is to transform the initial Hamiltonian $\mathcal{H}$ (with a large energy cutoff $\Lambda$ ) into an effective Hamiltonian $\mathcal{H}_{\lambda}$ which has no matrix elements belonging to transitions larger than $\lambda$. This is achieved by a unitary transformation so that the effective Hamiltonian will have the same eigenspectrum as the original Hamiltonian $\mathcal{H}$. However, as it will turn out, it is especially suitable to describe the low-energy excitations of the system. $\mathcal{H}_{\lambda}$ is defined by

$$
\mathcal{H}_{\lambda}=e^{X_{\lambda}} \mathcal{H} e^{-X_{\lambda}} .
$$

The generator $X_{\lambda}$ of the transformation has to be antiHermitian, $X_{\lambda}^{\dagger}=-X_{\lambda}$, so that $\mathcal{H}_{\lambda}$ is Hermitian for any $\lambda$. We look for an appropriate generator $X_{\lambda}$ so that $\mathcal{H}_{\lambda}$ has no matrix elements belonging to transitions larger than $\lambda$. This means that the following condition

$$
\mathbf{Q}_{\lambda} \mathcal{H}_{\lambda}=0
$$

has to be fulfilled. Equation (6) will be used below to specify $X_{\lambda}$.

The expression (5) for the effective Hamiltonian $\mathcal{H}_{\lambda}$ can we expanded with respect to $X_{\lambda}$

$$
\begin{aligned}
\mathcal{H}_{\lambda}= & \mathcal{H}+\left[X_{\lambda}, \mathcal{H}\right]+\frac{1}{2 !}\left[X_{\lambda},\left[X_{\lambda}, \mathcal{H}\right]\right] \\
& +\frac{1}{3 !}\left[X_{\lambda},\left[X_{\lambda},\left[X_{\lambda}, \mathcal{H}\right]\right]\right]+\ldots
\end{aligned}
$$

By assuming that $X_{\lambda}$ can be written as a power series in the perturbation parameter $\varepsilon$

$$
X_{\lambda}=\varepsilon X_{\lambda}^{(1)}+\varepsilon^{2} X_{\lambda}^{(2)}+\varepsilon^{3} X_{\lambda}^{(3)}+\ldots
$$

the effective Hamiltonian $\mathcal{H}_{\lambda}$ can be expanded in a power series in $\varepsilon$ as well

$$
\begin{aligned}
& \mathcal{H}_{\lambda}= \mathcal{H}_{0}+\varepsilon\left\{\mathcal{H}_{1}+\left[X_{\lambda}^{(1)}, \mathcal{H}_{0}\right]\right\} \\
&+\varepsilon^{2}\left\{\left[X_{\lambda}^{(1)}, \mathcal{H}_{1}\right]+\left[X_{\lambda}^{(2)}, \mathcal{H}_{0}\right]\right. \\
&\left.\quad+\frac{1}{2 !}\left[X_{\lambda}^{(1)},\left[X_{\lambda}^{(1)}, \mathcal{H}_{0}\right]\right]\right\}+\mathcal{O}\left(\varepsilon^{3}\right) .
\end{aligned}
$$

The different contributions $X_{\lambda}^{(n)}$ to the generator of the unitary transformation (5) can be successively determined from Eq. (6). One finds

$$
\begin{aligned}
\mathbf{Q}_{\lambda} X_{\lambda}^{(1)}= & \frac{1}{\mathbf{L}_{0}}\left(\mathbf{Q}_{\lambda} \mathcal{H}_{1}\right), \\
\mathbf{Q}_{\lambda} X_{\lambda}^{(2)}= & -\frac{1}{2 \mathbf{L}_{0}} \mathbf{Q}_{\lambda}\left[\left(\mathbf{Q}_{\lambda} \mathcal{H}_{1}\right), \frac{1}{\mathbf{L}_{0}}\left(\mathbf{Q}_{\lambda} \mathcal{H}_{1}\right)\right] \\
& -\frac{1}{\mathbf{L}_{0}} \mathbf{Q}_{\lambda}\left[\left(\mathbf{P}_{\lambda} \mathcal{H}_{1}\right), \frac{1}{\mathbf{L}_{0}}\left(\mathbf{Q}_{\lambda} \mathcal{H}_{1}\right)\right],
\end{aligned}
$$

and similar expression in higher order in $\varepsilon$. Here $\mathbf{L}_{0}$ denotes the unperturbed Liouville operator which is defined by $\mathbf{L}_{0} A=\left[\mathcal{H}_{0}, A\right]$ for any operator variable $A$. Note that by Eqs. (10), (11), and the equivalent equations for the higher orders in $\varepsilon$ the generators $X_{\lambda}^{(n)}$ are not completely fixed. The reason is that the parts $\mathbf{P}_{\lambda} X_{\lambda}^{(n)}$ of $X_{\lambda}^{(n)}$ are not fixed by Eq. (6) and can still be chosen arbitrarily. In the following we use for convenience

$$
\mathbf{P}_{\lambda} X_{\lambda}^{(1)}=\mathbf{P}_{\lambda} X_{\lambda}^{(2)}=0 .
$$

Inserting Eqs. (10) and (11) into Eq. (9) one finds for the effective Hamiltonian $\mathcal{H}_{\lambda}$ up to second order in $\mathcal{H}_{1}$

$$
\begin{aligned}
\mathcal{H}_{\lambda}= & \mathcal{H}_{0}+\mathbf{P}_{\lambda} \mathcal{H}_{1}-\frac{1}{2} \mathbf{P}_{\lambda}\left[\left(\mathbf{Q}_{\lambda} \mathcal{H}_{1}\right), \frac{1}{\mathbf{L}_{0}}\left(\mathbf{Q}_{\lambda} \mathcal{H}_{1}\right)\right] \\
& -\mathbf{P}_{\lambda}\left[\left(\mathbf{P}_{\lambda} \mathcal{H}_{1}\right), \frac{1}{\mathbf{L}_{0}}\left(\mathbf{Q}_{\lambda} \mathcal{H}_{1}\right)\right],
\end{aligned}
$$

where $\varepsilon$ was set equal to 1 . Note that the perturbation expansion (13) can easily be extended to higher orders in $\varepsilon$. One should also note that Eq. (13) automatically guarantees the correct size dependence of the Hamiltonian due to the commutators appearing in Eq. (13).

In the following section we will use Eq. (13) to establish a renormalization approach to many-particle systems by successively reducing the cutoff energy $\lambda$ to smaller and smaller values. In the limit $\lambda \rightarrow 0$ expression (13) provides an expression for an effective Hamiltonian that acts on a possibly degenerate ground-state of the unperturbed Hamiltonian. For this case one can also show that Eq. (13) reduces to an expression derived from usual perturbation theory [8]. A systematic extension of Eq. (13) to higher-order perturbation theory can also be used to provide a simple scheme for an algebraic evaluation of higher terms of $\mathcal{H}_{(\lambda=0)}$ by use of a computer [9]. Such a scheme does not require special properties of the eigenvalue spectrum of the unperturbed Hamiltonian $\mathcal{H}_{0}$. In particular, an equidistant spectrum is not needed. This property was implied for a perturbation expansion [10] based on Wegner's flow equation method.

\section{RENORMALIZATION APPROACH}

In this section we will discuss the elimination procedure for the interaction $\mathcal{H}_{1}$. However, instead of transforming the Hamiltonian in one step as was done in the preceding section the transformation will be performed successively. Or more formally spoken, instead of applying the elimination of high-energy excitations in one step a sequence of stepwise transformations is used in order to obtain an effectively diagonal model. In an infinitesimal formulation the renormalization of the coupling constants will be described by flow equations. In order to find these equations some approximations will 
be necessary. The goal of the method is to transform the initial Hamiltonian (with a large cutoff $\Lambda$ ) into an effective Hamiltonian which possesses only coupling terms between states with an energy difference less or equal to $\lambda$. Thereby, the method yields renormalization equations as function of the cutoff $\lambda$.

Let us start from the renormalized Hamiltonian

$$
\mathcal{H}_{\lambda}=\mathcal{H}_{0, \lambda}+\mathcal{H}_{1, \lambda}
$$

after all excitations with energy differences larger than $\lambda$ have been eliminated. In the next step an additional renormalization of $\mathcal{H}_{\lambda}$ to a new Hamiltonian $\mathcal{H}_{(\lambda-\Delta \lambda)}$ is done by eliminating all excitations within the energy shell between the cutoff $\lambda$ and a somewhat smaller energy cutoff $(\lambda-\Delta \lambda)$. The new Hamiltonian $\mathcal{H}_{(\lambda-\Delta \lambda)}$ will be calculated by use of the perturbation theory discussed above. One finds

$$
\begin{aligned}
& \mathcal{H}_{(\lambda-\Delta \lambda)}= \\
& =\mathbf{P}_{(\lambda-\Delta \lambda)} \mathcal{H}_{\lambda} \\
& \quad-\frac{1}{2} \mathbf{P}_{(\lambda-\Delta \lambda)}\left[\left(\mathbf{Q}_{(\lambda-\Delta \lambda)} \mathcal{H}_{1, \lambda}\right), \frac{1}{\mathbf{L}_{0, \lambda}}\left(\mathbf{Q}_{(\lambda-\Delta \lambda)} \mathcal{H}_{1, \lambda}\right)\right] \\
& \quad-\mathbf{P}_{(\lambda-\Delta \lambda)}\left[\left(\mathbf{P}_{(\lambda-\Delta \lambda)} \mathcal{H}_{1, \lambda}\right), \frac{1}{\mathbf{L}_{0, \lambda}}\left(\mathbf{Q}_{(\lambda-\Delta \lambda)} \mathcal{H}_{1, \lambda}\right)\right]
\end{aligned}
$$

$\mathbf{L}_{0, \lambda}$ is now the Liouville operator with respect to the $\lambda$ dependent unperturbed Hamiltonian $\mathcal{H}_{0, \lambda}$. As is obvious from Eq. (15) the second-order term on the right-hand side (rhs) may be divided into two parts. The first one connects eigenstates of $\mathcal{H}_{0, \lambda}$ with the same energy. This part commutes with $\mathcal{H}_{0, \lambda}$ and can therefore be considered as renormalization of the unperturbed Hamiltonian

$$
\begin{aligned}
& \mathcal{H}_{0,(\lambda-\Delta \lambda)}-\mathcal{H}_{0, \lambda}= \\
& -\frac{1}{2} \mathbf{P}_{0}\left[\left(\mathbf{Q}_{(\lambda-\Delta \lambda)} \mathcal{H}_{1, \lambda}\right), \frac{1}{\mathbf{L}_{0, \lambda}}\left(\mathbf{Q}_{(\lambda-\Delta \lambda)} \mathcal{H}_{1, \lambda}\right)\right] .
\end{aligned}
$$

In contrast the second part connects eigenstates of $\mathcal{H}_{0, \lambda}$ with different energies. It therefore represents a renormalization of the interaction part of the Hamiltonian

$$
\begin{aligned}
& \mathcal{H}_{1,(\lambda-\Delta \lambda)}-\mathbf{P}_{(\lambda-\Delta \lambda)} \mathcal{H}_{1, \lambda}= \\
& =-\mathbf{P}_{(\lambda-\Delta \lambda)}\left[\left(\mathbf{P}_{(\lambda-\Delta \lambda)} \mathcal{H}_{1, \lambda}\right), \frac{1}{\mathbf{L}_{0, \lambda}}\left(\mathbf{Q}_{(\lambda-\Delta \lambda)} \mathcal{H}_{1, \lambda}\right)\right] \\
& -\frac{1}{2}\left(\mathbf{P}_{(\lambda-\Delta \lambda)}-\mathbf{P}_{0}\right) \\
& \quad \times\left[\left(\mathbf{Q}_{(\lambda-\Delta \lambda)} \mathcal{H}_{1, \lambda}\right), \frac{1}{\mathbf{L}_{0, \lambda}}\left(\mathbf{Q}_{(\lambda-\Delta \lambda)} \mathcal{H}_{1, \lambda}\right)\right] \\
& \approx-\mathbf{P}_{(\lambda-\Delta \lambda)}\left[\left(\mathbf{P}_{(\lambda-\Delta \lambda)} \mathcal{H}_{1, \lambda}\right), \frac{1}{\mathbf{L}_{0, \lambda}}\left(\mathbf{Q}_{(\lambda-\Delta \lambda)} \mathcal{H}_{1, \lambda}\right)\right] .
\end{aligned}
$$

We assume that $\Delta \lambda$ is small. Therefore, only the mixed term, i.e., the second part on the rhs of Eq. (17), contributes to the renormalization of $\mathcal{H}_{1, \lambda}$. Thus, Eqs. (16) and (17) describe the renormalization of the unperturbed and of the interaction part of the Hamiltonian. They represent the main result of our theoretical formalism. In the limit $\Delta \lambda \rightarrow 0$, i.e. for vanishing shell width, these equation can be used to derive differential equations for the dependence of the Hamiltonian on the cutoff energy $\lambda$. The resulting equations for the parameters of the Hamiltonian are called flow equations. Their solution depend on the initial values of the parameters of the Hamiltonian and determine to final Hamiltonian in the limit $\lambda \rightarrow 0$. Note that for $\lambda \rightarrow 0$ the resulting Hamiltonian only consists of the unperturbed part $\mathcal{H}_{0,(\lambda \rightarrow 0)}$, so that an effectively diagonal Hamiltonian is obtained. The interaction $\mathcal{H}_{1,(\lambda \rightarrow 0)}$ completely vanishes since it is completely used up in the renormalization procedure. The detailed structure of the flow equations strongly depends on the system under consideration. However, one can easily write down these equations in matrix representation with respect to the unperturbed Hamiltonian (compare Appendix A).

Next we discuss the relation between the present renormalization approach and Wegner's flow equation method [1]. The approach presented here is also based on the idea to formulate flow equations for the Hamiltonian. However, there are substantial differences between both methods: i) The present approach starts directly from the renormalization of the Hamiltonian and not from continuous unitary transformations in differential form. ii) Moreover, the band diagonal form of the transformed Hamiltonian results from a physically motivated projection operator on low-energy transitions instead of introducing cutoff functions to restrict the Hamiltonian matrix. iii) The result of the renormalization approach (16) and (17) is directly formulated in terms of an operator relation. Wegner's flow equation approach [1] is usually written in matrix representation though it is also possible to give a formulation in terms of operators [5]. iv) As was discussed in Sec. II, there is a direct connection between the renormalization method and usual perturbation theory.

\section{THE EXACT SOLVABLE FANO-ANDERSON MODEL}

In this section we illustrate the renormalization approach for the case of a simple model, i.e., we apply the method to the exact solvable Fano-Anderson model $[6,7]$. This model is given by

$$
\begin{gathered}
\mathcal{H}=\mathcal{H}_{0}+\mathcal{H}_{1}, \\
\mathcal{H}_{0}=\varepsilon_{f} \sum_{i, m} f_{i m}^{\dagger} f_{i m}+\sum_{\mathbf{k}, m} \varepsilon_{\mathbf{k}} c_{\mathbf{k} m}^{\dagger} c_{\mathbf{k} m} \\
=\sum_{\mathbf{k}, m}\left(\varepsilon_{f} f_{\mathbf{k} m}^{\dagger} f_{\mathbf{k} m}+\varepsilon_{\mathbf{k}} c_{\mathbf{k} m}^{\dagger} c_{\mathbf{k} m}\right),
\end{gathered}
$$




$$
\begin{aligned}
\mathcal{H}_{1} & =\frac{1}{\sqrt{N}} \sum_{\mathbf{k}, i, m} V_{\mathbf{k}}\left(f_{i m}^{\dagger} c_{\mathbf{k} m} e^{\mathrm{i} \mathbf{k} \mathbf{R}_{i}}+\text { h.c. }\right) \\
& =\sum_{\mathbf{k}, m} V_{\mathbf{k}}\left(f_{\mathbf{k} m}^{\dagger} c_{\mathbf{k} m}+c_{\mathbf{k} m}^{\dagger} f_{\mathbf{k} m}\right) .
\end{aligned}
$$

A possible realization of the model is a periodic system of localized $f$ electrons which interact with conducting electrons thereby neglecting correlation effects. The index $i$ denotes the $f$ sites, $\mathbf{k}$ is the wave vector, and $V_{\mathbf{k}}$ describes the hybridization between conduction and localized electrons. The excitation energies $\varepsilon_{\mathbf{k}}$ and $\varepsilon_{f}$ for conduction and localized electrons are measured from the chemical potential $\mu$. As a further simplification, both types of electrons are assumed to have the same angular momentum index $m$ with values $m=1 \ldots \nu_{f}$. Of course, the model is easily solved and leads to two hybridized bands

$$
\begin{aligned}
\mathcal{H} & =\sum_{\mathbf{k}, m} \omega_{\mathbf{k}}^{(\alpha)} \alpha_{\mathbf{k} m}^{\dagger} \alpha_{\mathbf{k} m}+\sum_{\mathbf{k}, m} \omega_{\mathbf{k}}^{(\beta)} \beta_{\mathbf{k} m}^{\dagger} \beta_{\mathbf{k} m}, \\
\omega_{\mathbf{k}}^{(\alpha, \beta)} & =\frac{\varepsilon_{\mathbf{k}}+\varepsilon_{f}}{2} \pm \frac{1}{2} \sqrt{\left(\varepsilon_{\mathbf{k}}-\varepsilon_{f}\right)^{2}+4\left|V_{\mathbf{k}}\right|^{2}}
\end{aligned}
$$

with eigenmodes $\alpha_{\mathbf{k} m}^{\dagger}$ and $\beta_{\mathbf{k} m}^{\dagger}$ given by certain linear combinations of $c_{\mathbf{k} m}^{\dagger}$ and $f_{\mathbf{k} m}^{\dagger}$, i.e.,

$$
\begin{gathered}
\alpha_{\mathbf{k} m}^{\dagger}=u_{\mathbf{k}} f_{\mathbf{k} m}^{\dagger}+v_{\mathbf{k}} c_{\mathbf{k} m}^{\dagger}, \quad \beta_{\mathbf{k} m}^{\dagger}=-v_{\mathbf{k}} f_{\mathbf{k} m}^{\dagger}+u_{\mathbf{k}} c_{\mathbf{k} m}^{\dagger} \\
\left|u_{\mathbf{k}}\right|^{2}=\frac{1}{2}-\frac{1}{2} \frac{\varepsilon_{\mathbf{k}}-\varepsilon_{f}}{\sqrt{\left(\varepsilon_{\mathbf{k}}-\varepsilon_{f}\right)^{2}+4\left|V_{\mathbf{k}}\right|^{2}}} \\
\left|v_{\mathbf{k}}\right|^{2}=\frac{1}{2}+\frac{1}{2} \frac{\varepsilon_{\mathbf{k}}-\varepsilon_{f}}{\sqrt{\left(\varepsilon_{\mathbf{k}}-\varepsilon_{f}\right)^{2}+4\left|V_{\mathbf{k}}\right|^{2}}}
\end{gathered}
$$

In the renormalization approach we are integrating out particle-hole excitations of conduction and $f$ electrons described by the hybridization $\mathcal{H}_{1}$. We expect to obtain finally an effectively free model.

The starting point of the method is a renormalized Hamiltonian $\mathcal{H}_{\lambda}$ which is obtained after all excitations with energies larger than a given cutoff $\lambda$ have been eliminated. Due to the result of the preceeding section it should have the following form

$$
\begin{aligned}
\mathcal{H}_{\lambda} & =\mathcal{H}_{0, \lambda}+\mathcal{H}_{1, \lambda}, \\
\mathcal{H}_{0, \lambda} & =\sum_{\mathbf{k}, m}\left(\varepsilon_{\mathbf{k}, \lambda}^{f} f_{\mathbf{k} m}^{\dagger} f_{\mathbf{k} m}+\varepsilon_{\mathbf{k}, \lambda}^{c} c_{\mathbf{k} m}^{\dagger} c_{\mathbf{k} m}\right) \\
\mathcal{H}_{1, \lambda} & =\sum_{\substack{\mathbf{k}, m \\
\left|\varepsilon_{\mathbf{k}, \lambda}^{c}-\varepsilon_{\mathbf{k}, \lambda}^{f}\right| \leq \lambda}} V_{\mathbf{k}}\left(f_{\mathbf{k} m}^{\dagger} c_{\mathbf{k} m}+c_{\mathbf{k} m}^{\dagger} f_{\mathbf{k} m}\right)=\mathbf{P}_{\lambda} \mathcal{H}_{1} .
\end{aligned}
$$

As it turns out no renormalization of the hybridization $V_{\mathbf{k}}$ occurs so that $V_{\mathbf{k}}$ is assumed to be $\lambda$ independent from the beginning. However, the one-particle energies $\varepsilon_{\mathbf{k}, \lambda}^{c}$ and $\varepsilon_{\mathbf{k}, \lambda}^{f}$ will depend on the energy cutoff $\lambda$ and moreover $\varepsilon_{\mathbf{k}, \lambda}^{f}$ depend on the wave vector $\mathbf{k}$. Next, we evaluate the new Hamiltonian $\mathcal{H}_{0,(\lambda-\Delta \lambda)}$ by projecting out all excitations between $\lambda-\Delta \lambda$ and $\lambda$ [compare (16)]. By inserting Eq. (21) into Eq. (16) we obtain

$$
\begin{aligned}
& \delta \mathcal{H}_{0}(\lambda, \Delta \lambda)=\mathcal{H}_{0,(\lambda-\Delta \lambda)}-\mathcal{H}_{0, \lambda} \\
& =\sum_{\mathbf{k}, m}^{\prime}\left\{-\frac{\left|V_{\mathbf{k}}\right|^{2}}{\varepsilon_{\mathbf{k}, \lambda}^{c}-\varepsilon_{\mathbf{k}, \lambda}^{f}}+\ldots\right\}\left[f_{\mathbf{k} m}^{\dagger} f_{\mathbf{k} m}-c_{\mathbf{k} m}^{\dagger} c_{\mathbf{k} m}\right]
\end{aligned}
$$

where the Fermion commutation relations were used. The prime / above the sum indicates that the condition $(\lambda-\Delta \lambda)<\left|\varepsilon_{\mathbf{k}, \lambda}^{c}-\varepsilon_{\mathbf{k}, \lambda}^{f}\right| \leq \lambda$ has to be fulfilled. Note that in Eq. (22) only second-order contributions are explicitly given. Higher-order terms are indicated by dots ... . The renormalization equations for the one-particle energies are easily found from Eq. (22) and read

$$
\begin{aligned}
& \varepsilon_{\mathbf{k},(\lambda-\Delta \lambda)}^{f}-\varepsilon_{\mathbf{k}, \lambda}^{f}=\left\{-\frac{\left|V_{\mathbf{k}}\right|^{2}}{\varepsilon_{\mathbf{k}, \lambda}^{c}-\varepsilon_{\mathbf{k}, \lambda}^{f}}+\ldots\right\} \Theta(\lambda, \Delta \lambda), \\
& \varepsilon_{\mathbf{k},(\lambda-\Delta \lambda)}^{c}-\varepsilon_{\mathbf{k}, \lambda}^{c}=-\left\{-\frac{\left|V_{\mathbf{k}}\right|^{2}}{\varepsilon_{\mathbf{k}, \lambda}^{c}-\varepsilon_{\mathbf{k}, \lambda}^{f}}+\ldots\right\} \Theta(\lambda, \Delta \lambda)
\end{aligned}
$$

where

$$
\begin{aligned}
\Theta(\lambda, \Delta \lambda)= & \Theta\left(\left|\varepsilon_{\mathbf{k}, \lambda}^{c}-\varepsilon_{\mathbf{k}, \lambda}^{f}\right|-(\lambda-\Delta \lambda)\right) \\
& -\Theta\left(\left|\varepsilon_{\mathbf{k}, \lambda}^{c}-\varepsilon_{\mathbf{k}, \lambda}^{f}\right|-\lambda\right) .
\end{aligned}
$$

To solve these equations we subtract Eq. (23) from Eq. (24), multiply both sides by $\left(\varepsilon_{\mathbf{k}, \lambda}^{c}-\varepsilon_{\mathbf{k}, \lambda}^{f}\right)$, and divide by $\Delta \lambda$. By performing the limit $\Delta \lambda \rightarrow 0$ we obtain

$$
\frac{d}{d \lambda}\left(\varepsilon_{\mathbf{k}, \lambda}^{c}-\varepsilon_{\mathbf{k}, \lambda}^{f}\right)^{2}=-4\left|V_{\mathbf{k}}\right|^{2} \lim _{\Delta \lambda \rightarrow 0} \frac{\Theta(\lambda, \Delta \lambda)}{\Delta \lambda} .
$$

Note that Eq. (26) includes all renormalization contributions of higher orders in the interaction. It turns out that the solution of Eq. (26) shows a step like behavior and reads

$$
\begin{aligned}
& \left|\varepsilon_{\mathbf{k}, \lambda}^{c}-\varepsilon_{\mathbf{k}, \lambda}^{f}\right|=\left|\varepsilon_{\mathbf{k}}-\varepsilon_{f}\right|+C_{\mathbf{k}} \Theta\left(\left|\varepsilon_{\mathbf{k}}-\varepsilon_{f}\right|-\lambda\right) \\
& \text { with } \quad C_{\mathbf{k}}=\sqrt{\left(\varepsilon_{\mathbf{k}}-\varepsilon_{f}\right)^{2}+4\left|V_{\mathbf{k}}\right|^{2}}-\left|\varepsilon_{\mathbf{k}}-\varepsilon_{f}\right| .
\end{aligned}
$$

To prove this result one has to insert Eq. (27) into Eq. (26) which gives $-4\left|V_{\mathbf{k}}\right|^{2} \delta\left(\left|\varepsilon_{\mathbf{k}}-\varepsilon_{f}\right|-\lambda\right)$ for both sides. Note that the evaluation of the rhs. of Eq. (26) together with Eq. (25) does not give $-4\left|V_{\mathbf{k}}\right|^{2} \delta\left(\left|\varepsilon_{\mathbf{k}, \lambda}^{c}-\varepsilon_{\mathbf{k}, \lambda}^{f}\right|-\lambda\right)$ as would be expected of first glance. This follows from the step like renormalization of $\left|\varepsilon_{\mathbf{k}, \lambda}^{c}-\varepsilon_{\mathbf{k}, \lambda}^{f}\right|$, described by Eq. (27).

For $\lambda \rightarrow 0$ one finds 


$$
\left(\tilde{\varepsilon}_{\mathbf{k}}^{c}-\tilde{\varepsilon}_{\mathbf{k}}^{f}\right)^{2}=\left(\varepsilon_{\mathbf{k}}-\varepsilon_{f}\right)^{2}+4\left|V_{\mathbf{k}}\right|^{2}
$$

where $\tilde{\varepsilon}_{\mathbf{k}}^{c}$ and $\tilde{\varepsilon}_{\mathbf{k}}^{f}$ denote the one-particle energies at $\lambda \rightarrow 0$,

$$
\tilde{\varepsilon}_{\mathbf{k}}^{c}=\varepsilon_{\mathbf{k},(\lambda \rightarrow 0)}^{c}, \quad \tilde{\varepsilon}_{\mathbf{k}}^{f}=\varepsilon_{\mathbf{k},(\lambda \rightarrow 0)}^{f} .
$$

On the other hand, it is easily seen that the summation of Eqs. (23) and (24) leads to

$$
\tilde{\varepsilon}_{\mathbf{k}}^{c}+\tilde{\varepsilon}_{\mathbf{k}}^{f}=\varepsilon_{\mathbf{k}}+\varepsilon_{f} .
$$

The system of Eqs. (28) and (30) can easily be solved and gives

$$
\begin{aligned}
& \tilde{\varepsilon}_{\mathbf{k}}^{c}=\frac{\varepsilon_{\mathbf{k}}+\varepsilon_{f}}{2}+\frac{1}{2} \sqrt{\left(\varepsilon_{\mathbf{k}}-\varepsilon_{f}\right)^{2}+4\left|V_{\mathbf{k}}\right|^{2}}, \\
& \tilde{\varepsilon}_{\mathbf{k}}^{f}=\frac{\varepsilon_{\mathbf{k}}+\varepsilon_{f}}{2}-\frac{1}{2} \sqrt{\left(\varepsilon_{\mathbf{k}}-\varepsilon_{f}\right)^{2}+4\left|V_{\mathbf{k}}\right|^{2}} .
\end{aligned}
$$

Note that Eq. (32) agrees with the exact solution Eq. (19). As a result we have obtained the following free model

$$
\mathcal{H}_{(\lambda \rightarrow 0)}=\sum_{\mathbf{k}, m}\left(\tilde{\varepsilon}_{\mathbf{k}}^{c} c_{\mathbf{k} m}^{\dagger} c_{\mathbf{k} m}+\tilde{\varepsilon}_{\mathbf{k}}^{f} f_{\mathbf{k} m}^{\dagger} f_{\mathbf{k} m}\right) .
$$

Note that the interaction $\mathcal{H}_{1, \lambda}$ vanishes for $\lambda \rightarrow 0$ since it is completely used to renormalize the one-particle energies $\widetilde{\varepsilon}_{\mathbf{k}}^{c}$ and $\tilde{\varepsilon}_{\mathbf{k}}^{f}$. The result Eqs. (33) and (32) can be used to evaluate expectation values. Since $\mathcal{H}_{(\lambda \rightarrow 0)}$ emerged from the original model $\mathcal{H}$ by an unitary transformation, the free energie can also be calculated from $\mathcal{H}_{(\lambda \rightarrow 0)}$

$$
F=-\frac{1}{\beta} \ln \operatorname{Tr} e^{-\beta \mathcal{H}}=-\frac{1}{\beta} \ln \operatorname{Tr} e^{-\beta \mathcal{H}_{(\lambda \rightarrow 0)}}=: F_{(\lambda \rightarrow 0)} .
$$

Then, the $f$ electron occupation number is found from $F_{(\lambda \rightarrow 0)}$ by functional derivative

$$
\begin{aligned}
\left\langle f_{\mathbf{k} m}^{\dagger} f_{\mathbf{k} m}\right\rangle= & \frac{1}{N} \frac{\partial F}{\partial \varepsilon_{f}}=\frac{1}{N} \frac{\partial F_{(\lambda \rightarrow 0)}}{\partial \varepsilon_{f}} \\
= & \frac{1}{1+e^{\beta \tilde{\varepsilon}_{\mathbf{k}}^{f}}}\left(\frac{1}{2}+\frac{1}{2} \frac{\varepsilon_{\mathbf{k}}-\varepsilon_{f}}{\sqrt{\left(\varepsilon_{\mathbf{k}}-\varepsilon_{f}\right)^{2}+4\left|V_{\mathbf{k}}\right|^{2}}}\right) \\
& +\frac{1}{1+e^{\beta \tilde{\varepsilon}_{\mathbf{k}}^{c}}}\left(\frac{1}{2}-\frac{1}{2} \frac{\varepsilon_{\mathbf{k}}-\varepsilon_{f}}{\sqrt{\left(\varepsilon_{\mathbf{k}}-\varepsilon_{f}\right)^{2}+4\left|V_{\mathbf{k}}\right|^{2}}}\right)
\end{aligned}
$$

where Eqs. (32) and (34) was used. On the other hand, static and dynamic expectation values can be evaluated by applying the unitary transformations not only on the Hamiltonian but on all operator quantities which appear in expectation values. By exploiting that operator expressions under a trace are invariant against unitary transformations the $f$ electron occupation number is also given by

$$
\begin{aligned}
\left\langle f_{\mathbf{k} m}^{\dagger} f_{\mathbf{k} m}\right\rangle & =\left\langle f_{\mathbf{k} m}^{\dagger}(\lambda) f_{\mathbf{k} m}(\lambda)\right\rangle_{\lambda} \\
& =\left\langle f_{\mathbf{k} m}^{\dagger}(\lambda \rightarrow 0) f_{\mathbf{k} m}(\lambda \rightarrow 0)\right\rangle_{(\lambda \rightarrow 0)}
\end{aligned}
$$

where

$$
f_{\mathbf{k} m}^{\dagger}(\lambda)=e^{X_{\lambda}} f_{\mathbf{k} m}^{\dagger} e^{-X_{\lambda}}
$$

Here, $\langle\ldots\rangle_{\lambda}$ means the expectation value formed with $\mathcal{H}_{\lambda}$. One possible way to proceed is to derive flow equations for the $\lambda$-dependent operators $f_{\mathbf{k} m}^{\dagger}(\lambda), f_{\mathbf{k} m}(\lambda)$. However, this may lead to inconsistent approximations which are necessary to solve the obtained flow equations. On the other hand, this problem can be avoided by using an appropriate ansatz for the $\lambda$-dependence. Therefore, for the $\lambda$-dependent operators $f_{\mathbf{k} m}^{\dagger}(\lambda)$ and for the second operator $c_{\mathbf{k} m}^{\dagger}(\lambda)$ we shall use

$$
\begin{aligned}
& f_{\mathbf{k} m}^{\dagger}(\lambda)=u_{\mathbf{k}, \lambda} f_{\mathbf{k} m}^{\dagger}+v_{\mathbf{k}, \lambda} c_{\mathbf{k} m}^{\dagger} \quad \text { and } \\
& c_{\mathbf{k} m}^{\dagger}(\lambda)=-v_{\mathbf{k}, \lambda} f_{\mathbf{k} m}^{\dagger}+u_{\mathbf{k}, \lambda} c_{\mathbf{k} m}^{\dagger}
\end{aligned}
$$

with $\left|u_{\mathbf{k}, \lambda}\right|^{2}+\left|v_{\mathbf{k}, \lambda}\right|^{2}=1$. This form is suggested by repeatedly applying the unitary transformation on $f_{\mathbf{k} m}^{\dagger}$ and $c_{\mathbf{k} m}^{\dagger}$. With Eq. (38) the expectation value $\left\langle f_{\mathbf{k} m}^{\dagger} f_{\mathbf{k} m}\right\rangle$ can easily be evaluated. One finds

$$
\begin{aligned}
\left\langle f_{\mathbf{k} m}^{\dagger} f_{\mathbf{k} m}\right\rangle & =\left\langle f_{\mathbf{k} m}^{\dagger}(\lambda \rightarrow 0) f_{\mathbf{k} m}(\lambda \rightarrow 0)\right\rangle_{(\lambda \rightarrow 0)} \\
& =\left|u_{\mathbf{k},(\lambda \rightarrow 0)}\right|^{2} \frac{1}{1+e^{\beta \tilde{\varepsilon}_{\mathbf{k}}^{f}}}+\left|v_{\mathbf{k},(\lambda \rightarrow 0)}\right|^{2} \frac{1}{1+e^{\beta \tilde{\varepsilon}_{\mathbf{k}}^{c}}} .
\end{aligned}
$$

If one compares both results for $\left\langle f_{\mathbf{k} m}^{\dagger} f_{\mathbf{k} m}\right\rangle$ the prefactors $u_{\mathbf{k},(\lambda \rightarrow 0)}$ and $u_{\mathbf{k},(\lambda \rightarrow 0)}$ can be found

$$
\begin{aligned}
& \left|u_{\mathbf{k},(\lambda \rightarrow 0)}\right|^{2}=\frac{1}{2}+\frac{1}{2} \frac{\varepsilon_{\mathbf{k}}-\varepsilon_{f}}{\sqrt{\left(\varepsilon_{\mathbf{k}}-\varepsilon_{f}\right)^{2}+4\left|V_{\mathbf{k}}\right|^{2}}}, \\
& \left|v_{\mathbf{k},(\lambda \rightarrow 0)}\right|^{2}=\frac{1}{2}-\frac{1}{2} \frac{\varepsilon_{\mathbf{k}}-\varepsilon_{f}}{\sqrt{\left(\varepsilon_{\mathbf{k}}-\varepsilon_{f}\right)^{2}+4\left|V_{\mathbf{k}}\right|^{2}}} .
\end{aligned}
$$

This result agrees with Eq. (20). Thus we have obtained the exact transformations for the $f$ and $c$ operators. It follows that all static and and dynamic quantities involving electron creation and annihilation operators can be calculated in the framework of the renormalization approach. 


\section{APPLICATION TO PERIODIC ANDERSON MODEL}

In the following section we will refer to the Anderson lattice model [6] which describes an interacting system of conduction electrons and correlated localized $4 f$ electrons, arranged periodically on a lattice. Within a simplified version the Hamiltonian of the model can be written as

$$
\begin{aligned}
\mathcal{H} & =\mathcal{H}_{0}+\mathcal{H}_{1}, \\
\mathcal{H}_{0} & =\varepsilon_{f} \sum_{i, m} \hat{f}_{i m}^{\dagger} \hat{f}_{i m}+\sum_{\mathbf{k}, m} \varepsilon_{\mathbf{k}} c_{\mathbf{k} m}^{\dagger} c_{\mathbf{k} m}, \\
\mathcal{H}_{1} & =\frac{1}{\sqrt{N}} \sum_{\mathbf{k}, i, m} V_{\mathbf{k}}\left(\hat{f}_{i m}^{\dagger} c_{\mathbf{k} m} e^{\mathrm{i} \mathbf{k} \mathbf{R}_{i}}+\text { h.c. }\right) .
\end{aligned}
$$

Here, $i$ is the $4 f$ site index, $\mathbf{k}$ is the conduction electron wave vector, and $V_{\mathbf{k}}$ is the hybridization matrix element between conduction and localized electrons. $\varepsilon_{f}$ and $\varepsilon_{\mathbf{k}}$, both measured from the chemical potential $\mu$, are the excitation energies for $4 f$ and conduction electrons, respectively. As a simplification, both types of electrons are assumed to have the same angular momentum index $m$ with $\nu_{f}$ values, $m=1 \ldots \nu_{f}$. This will make it possible to classify terms in an $1 / \nu_{f}$ expansion as is well known from the one-impurity model [11]. Finally, the local Coulomb repulsion $U_{f}$ at the $4 f$ sites has been assumed to be infinitely large so that localized sites can either be empty or singly occupied, i.e., the $\hat{f}_{i m}^{\dagger}$ is defined by

$$
\hat{f}_{i m}^{\dagger}=f_{i m}^{\dagger} \prod_{\tilde{m}(\neq m)}\left(1-n_{i \tilde{m}}^{f}\right) .
$$

\section{A. Perturbation theory}

For the model (41) we first evaluate the effective Hamiltonian $\mathcal{H}_{\lambda}$ in perturbation theory. In contrast to the general discussion in Sec. II now we are only interested in contributions which renormalize the unperturbed part $\mathcal{H}_{0}$ of the Hamiltonian. Using Eq. (13) one finds

$$
\begin{aligned}
\delta \mathcal{H}_{0, \lambda} & =\mathcal{H}_{0, \lambda}-\mathcal{H}_{0} \\
& =-\frac{1}{N} \sum_{\substack{i, \mathbf{k}, m \\
\left|\varepsilon_{\mathbf{k}}-\varepsilon_{f}\right|>\lambda}} \frac{\left|V_{\mathbf{k}}\right|^{2}}{\varepsilon_{\mathbf{k}}-\varepsilon_{f}}\left[\hat{f}_{i m}^{\dagger} c_{\mathbf{k} m}, c_{\mathbf{k} m}^{\dagger} \hat{f}_{i m}\right]
\end{aligned}
$$

Note that contributions which involve different $f$ sites have been neglected for simplicity. In the following we restrict ourselves to the dominant order in $\nu_{f}$ so that Eq. (43) reads

$$
\begin{aligned}
\delta \mathcal{H}_{0, \lambda}= & \frac{1}{N} \sum_{\substack{i, \mathbf{k}, m \\
\left|\varepsilon_{\mathbf{k}}-\varepsilon_{f}\right|>\lambda}} \frac{\left|V_{\mathbf{k}}\right|^{2}}{\varepsilon_{\mathbf{k}}-\varepsilon_{f}}\left\{\left\langle\hat{f}_{i m} \hat{f}_{i m}^{\dagger}\right\rangle c_{\mathbf{k} m}^{\dagger} c_{\mathbf{k} m}+\right. \\
& \left.+\left\langle c_{\mathbf{k} m}^{\dagger} c_{\mathbf{k} m}\right\rangle \hat{f}_{i m} \hat{f}_{i m}^{\dagger}+-\left\langle c_{\mathbf{k} m}^{\dagger} c_{\mathbf{k} m}\right\rangle\left\langle\hat{f}_{i m} \hat{f}_{i m}^{\dagger}\right\rangle\right\} .
\end{aligned}
$$

The restriction to contributions of dominant order in $\nu_{f}$ leads to a partial suppression of charge fluctuations on $f$ sites. The contributions, taken into account in Eq. (44), describe first the creation of an $f$ and the annihilation of an $c$-electron followed by the inverse process. Therefore, the renormalization contributions in dominant order in $\nu_{f}$ are based on the existence of a finite probability for empty $f$ sites. Furthermore, in Eq. (44) a factorization with respect to the full Hamiltonian $\mathcal{H}$ was carried out. Note that a factorization with respect to $\mathcal{H}$ instead of with $\mathcal{H}_{\lambda}$ is suggested by the following reason: As discussed in Sec. II, $\mathcal{H}_{\lambda}$ results from an unitary transformation which is applied to the full Hamiltonian $\mathcal{H}$. Expectation values are not changed by an unitary transformation if both operators and the Hamiltonian are transformed. Therefore, the factorization with respect to the original Hamiltonian $\mathcal{H}$ corresponds to taking into account higher-order renormalization.

Equation (44) can be simplified if we introduce the projection operator on an empty $f$ state at site $i$

$$
\begin{aligned}
\mathcal{P}_{0}(i) & =\prod_{\tilde{m}}\left(1-n_{i \tilde{m}}^{f}\right)=\hat{f}_{i m} \hat{f}_{i m}^{\dagger} \\
& =1-\sum_{\tilde{m}} \hat{f}_{i \tilde{m}}^{\dagger} \hat{f}_{i \tilde{m}}
\end{aligned}
$$

(independent of $m$ ). Note that the last equation exploits the completeness relation on an $f$ site. Here, the $f$ occupation number operator, $n_{i}^{f}=\sum_{\tilde{m}} \hat{f}_{i \tilde{m}}^{\dagger} \hat{f}_{i \tilde{m}}$, is at the same time the projection on the singly occupied $f$ site $i$. According to Eq. (44) the renormalization of the unperturbed Hamiltonian $\mathcal{H}_{0}$ can be written up to second order as

$$
\begin{aligned}
\delta \mathcal{H}_{0, \lambda}= & \delta \varepsilon_{f, \lambda} \sum_{i, m} \hat{f}_{i m}^{\dagger} \hat{f}_{i m}+\sum_{\mathbf{k}, m} \delta \varepsilon_{\mathbf{k}, \lambda} c_{\mathbf{k} m}^{\dagger} c_{\mathbf{k} m}+\delta E_{\lambda}, \\
\delta \varepsilon_{f, \lambda}= & -\frac{\nu_{f}}{N} \sum_{\mathbf{k}} \frac{\left|V_{\mathbf{k}}\right|^{2}}{\varepsilon_{\mathbf{k}}-\varepsilon_{f}}\left\langle n_{\mathbf{k} m}^{c}\right\rangle \Theta\left(\left|\varepsilon_{\mathbf{k}}-\varepsilon_{f}\right|-\lambda\right), \\
\delta \varepsilon_{\mathbf{k}, \lambda}= & \frac{1}{N} \sum_{i} \frac{\left|V_{\mathbf{k}}\right|^{2}}{\varepsilon_{\mathbf{k}}-\varepsilon_{f}}\left\langle\mathcal{P}_{0}(i)\right\rangle \Theta\left(\left|\varepsilon_{\mathbf{k}}-\varepsilon_{f}\right|-\lambda\right), \\
\delta E_{\lambda}= & \frac{\nu_{f}}{N} \sum_{i, \mathbf{k}} \frac{\left|V_{\mathbf{k}}\right|^{2}}{\varepsilon_{\mathbf{k}}-\varepsilon_{f}}\left\langle n_{\mathbf{k} m}^{c}\right\rangle\left(1-\left\langle\mathcal{P}_{0}(i)\right\rangle\right) \\
& \times \Theta\left(\left|\varepsilon_{\mathbf{k}}-\varepsilon_{f}\right|-\lambda\right),
\end{aligned}
$$

where $\delta \varepsilon_{\mathbf{k}, \lambda}$ and $\delta \varepsilon_{f, \lambda}$ lead to the renormalization of the one-particle energies $\varepsilon_{\mathbf{k}, \lambda}$ and $\varepsilon_{f, \lambda} . \delta E_{\lambda}$ is an additional energy shift. 


\section{B. Renormalization equations}

The above result in perturbation can be used to derive flow equations for the periodic Anderson model along the line discussed in Sec. III. Let us start by formally writing down the effective Hamiltonian $\mathcal{H}_{\lambda}$ after all excitations with energy differences larger than $\lambda$ have been eliminated. $\mathcal{H}_{\lambda}$ should have the following form

$$
\begin{aligned}
\mathcal{H}_{\lambda} & =\mathcal{H}_{0, \lambda}+\mathcal{H}_{1, \lambda} \\
\mathcal{H}_{0, \lambda} & =\varepsilon_{f, \lambda} \sum_{i, m} \hat{f}_{i m}^{\dagger} \hat{f}_{i m}+\sum_{\mathbf{k}, m} \varepsilon_{\mathbf{k}, \lambda} c_{\mathbf{k} m}^{\dagger} c_{\mathbf{k} m}+E_{\lambda} \\
\mathcal{H}_{1, \lambda} & =\mathbf{P}_{\lambda} \mathcal{H}_{1} \\
& =\frac{1}{\sqrt{N}} \sum_{\substack{i, \mathbf{k}, m \\
\left|\varepsilon_{\mathbf{k}, \lambda}-\varepsilon_{f, \lambda}\right| \leq \lambda}} V_{\mathbf{k}}\left(\hat{f}_{i m}^{\dagger} c_{\mathbf{k} m} e^{i \mathbf{k} \mathbf{R}_{i}}+\text { h.c. }\right)
\end{aligned}
$$

In the following we are only interested in the renormalization of the diagonal part of the Hamiltonian. Consequently, all additional terms which would appear due to Eqs. (16) and (17) will be neglected in the following for simplicity. Due to renormalization processes the oneparticle energies $\varepsilon_{f, \lambda}, \varepsilon_{\mathbf{k}, \lambda}$, as well as the energy shift $E_{\lambda}$ now depend on the energy cutoff $\lambda$. The initial conditions are those from the original model

$$
\varepsilon_{f,(\lambda=\Lambda)}=\varepsilon_{f}, \quad \varepsilon_{\mathbf{k},(\lambda=\Lambda)}=\varepsilon_{\mathbf{k}}, \quad E_{(\lambda=\Lambda)}=0 .
$$

In the following step let us evaluate a new effective Hamiltonian which is obtained after a further elimination of excitations within a small energy shell between $\lambda-\Delta \lambda$ and $\lambda$ has been done. The new Hamiltonian $\mathcal{H}_{(\lambda-\Delta \lambda)}$ is obtained from Eq. (46) and possesses the following renormalized parameters

$$
\begin{aligned}
\varepsilon_{f,(\lambda-\Delta \lambda)}= & \varepsilon_{f, \lambda}-\frac{\nu_{f}}{N} \sum_{\mathbf{k}}^{\prime} \frac{\left|V_{\mathbf{k}}\right|^{2}}{\varepsilon_{\mathbf{k}, \lambda}-\varepsilon_{f, \lambda}}\left\langle n_{\mathbf{k} m}^{c}\right\rangle, \\
\varepsilon_{\mathbf{k},(\lambda-\Delta \lambda)}= & \varepsilon_{\mathbf{k}, \lambda}+\frac{1}{N} \sum_{i}^{\prime} \frac{\left|V_{\mathbf{k}}\right|^{2}}{\varepsilon_{\mathbf{k}, \lambda}-\varepsilon_{f, \lambda}}\left\langle\mathcal{P}_{0}(i)\right\rangle, \\
E_{(\lambda-\Delta \lambda)}= & E_{\lambda}+\frac{\nu_{f}}{N} \sum_{i, \mathbf{k}}^{\prime} \frac{\left|V_{\mathbf{k}}\right|^{2}}{\varepsilon_{\mathbf{k}, \lambda}-\varepsilon_{f, \lambda}}\left\langle n_{\mathbf{k} m}^{c}\right\rangle \\
& \times\left(1-\left\langle\mathcal{P}_{0}(i)\right\rangle\right) .
\end{aligned}
$$

Here, the prime / above the sums indicates that the restriction $(\lambda-\Delta \lambda)<\left|\varepsilon_{\mathbf{k}, \lambda}-\varepsilon_{f, \lambda}\right| \leq \lambda$ has to be fulfilled.

Finally, we would like to perform the stepwise transformation continuously. By taking the limit $\Delta \lambda \rightarrow 0$, we obtain the following flow equations for the parameter $\varepsilon_{f, \lambda}, \varepsilon_{\mathbf{k}, \lambda}$, and $E_{\lambda}$

$$
\begin{aligned}
\frac{d \varepsilon_{f, \lambda}}{d \lambda}= & \frac{\nu_{f}}{N} \sum_{\mathbf{k}} \frac{\left|V_{k}\right|^{2}}{\varepsilon_{\mathbf{k}, \lambda}-\varepsilon_{f, \lambda}}\left\langle n_{\mathbf{k} m}^{c}\right\rangle \delta\left(\lambda-\left|\varepsilon_{\mathbf{k}, \lambda}-\varepsilon_{f, \lambda}\right|\right) \\
\frac{d \varepsilon_{\mathbf{k}, \lambda}}{d \lambda}= & -\frac{\left|V_{k}\right|^{2}}{\varepsilon_{\mathbf{k}, \lambda}-\varepsilon_{f, \lambda}}\left\langle\mathcal{P}_{0}(i)\right\rangle \delta\left(\lambda-\left|\varepsilon_{\mathbf{k}, \lambda}-\varepsilon_{f, \lambda}\right|\right) \\
\frac{d E_{\lambda}}{d \lambda}= & -\nu_{f} \sum_{\mathbf{k}} \frac{|V(k)|^{2}}{\varepsilon_{\mathbf{k}, \lambda}-\varepsilon_{f, \lambda}}\left\langle n_{\mathbf{k} m}^{c}\right\rangle\left(1-\left\langle\mathcal{P}_{0}(i)\right\rangle\right) \\
& \left.\times \delta\left(\lambda-\left|\varepsilon_{\mathbf{k}, \lambda}-\varepsilon_{f, \lambda}\right|\right)\right) \\
= & -N\left(\left\langle 1-\mathcal{P}_{0}(i)\right\rangle\right) \frac{d \varepsilon_{f, \lambda}}{d \lambda}
\end{aligned}
$$

Here, due to the $\mathbf{k}$ summation in Eq. (50) it was assumed that the renormalization of the one-particle energies $\varepsilon_{f, \lambda}$ and $\varepsilon_{\mathbf{k}, \lambda}$ is continuous in contrast to the case of Sec. IV. The first two equations are coupled nonlinear differential equations for the parameters $\varepsilon_{f, \lambda}$ and $\varepsilon_{\mathbf{k}, \lambda}$. Here, the $\delta$-function guarantees that only excitation on the energy shell contribute.

To solve Eqs. (50) and (51) first note that due to translational invariance the expectation value $\left\langle\mathcal{P}_{0}(i)\right\rangle$ is independent of site index $i$. Therefore, a simple relation between $\varepsilon_{f, \lambda}$ and $\varepsilon_{\mathbf{k}, \lambda}$ can be derived by inserting (51) into $(50)$

$$
\left\langle\mathcal{P}_{0}(i)\right\rangle \frac{d \varepsilon_{f, \lambda}}{d \lambda}=-\frac{\nu_{f}}{N} \sum_{\mathbf{k}}\left\langle n_{\mathbf{k} m}^{c}\right\rangle \frac{d \varepsilon_{\mathbf{k}, \lambda}}{d \lambda} .
$$

Its integration between the lower cutoff $\lambda \rightarrow 0$ and the cutoff $\Lambda$ of the original model leads to

$$
\left\langle\mathcal{P}_{0}(i)\right\rangle\left(\varepsilon_{f}-\tilde{\varepsilon}_{f}\right)=-\frac{\nu_{f}}{N} \sum_{\mathbf{k}}\left\langle n_{\mathbf{k} m}^{c}\right\rangle\left(\varepsilon_{\mathbf{k}}-\tilde{\varepsilon}_{\mathbf{k}}\right)
$$

where we have defined $\tilde{\varepsilon}_{f}=\varepsilon_{f,(\lambda \rightarrow 0)}$ and $\tilde{\varepsilon}_{k}=\varepsilon_{\mathbf{k},(\lambda \rightarrow 0)}$. Note that as before the expectation values in Eq. (54) are assumed to be independent of $\lambda$ since they were defined with the full Hamiltonian. Eq.(54) is the first equation which relates $\tilde{\varepsilon}_{f}$ and $\tilde{\varepsilon}_{k}$. To find a second equation let us integrate Eq. (51) between the lower cutoff $(\lambda \rightarrow 0)$ and the cutoff $\Lambda$ of the original model. One finds

$$
\begin{aligned}
\tilde{\varepsilon}_{\mathbf{k}}= & \varepsilon_{k}+\int_{(\lambda \rightarrow 0)}^{\Lambda} d \lambda^{\prime} \frac{\left|V_{k}\right|^{2}\left\langle\mathcal{P}_{0}(i)\right\rangle}{\varepsilon_{\mathbf{k}, \lambda^{\prime}}-\varepsilon_{f, \lambda^{\prime}}} \\
& \times \delta\left(\lambda^{\prime}-\left|\varepsilon_{\mathbf{k}, \lambda^{\prime}}-\varepsilon_{f, \lambda^{\prime}}\right|\right)
\end{aligned}
$$

where Eq. (48) was used. Since the flow of the oneparticle energies $\varepsilon_{\mathbf{k}, \lambda}$ and $\varepsilon_{f, \lambda}$ was assumed to be continous, the main contribution to the integral in Eq. (55) should originate from small values of the denominators $\varepsilon_{\mathbf{k}, \lambda^{\prime}}-\varepsilon_{f, \lambda^{\prime}}$, i.e., from small values of $\lambda^{\prime}$. Therefore, a reasonable approximation is to replace the quantity $\varepsilon_{\mathbf{k}, \lambda^{\prime}}-\varepsilon_{f, \lambda^{\prime}}$ in Eq. (55) by its value at the lower cutoff $\lambda^{\prime} \approx \lambda \rightarrow 0$. Thus we obtain

$$
\tilde{\varepsilon}_{\mathbf{k}}=\varepsilon_{\mathbf{k}}+\frac{\left|V_{k}\right|^{2}\left\langle\mathcal{P}_{0}(i)\right\rangle}{\tilde{\varepsilon}_{\mathbf{k}}-\tilde{\varepsilon}_{f}} .
$$


Note that additional contributions of higher order in $\left|V_{\mathbf{k}}\right|^{2}$ have been neglected.

Equations (56) and (54) fix the one-particle energies. The Hamiltonian $\mathcal{H}_{(\lambda \rightarrow 0)}$ reads

$$
\mathcal{H}_{(\lambda \rightarrow 0)}=\tilde{\varepsilon}_{f} \sum_{i, m} \hat{f}_{i m}^{\dagger} \hat{f}_{i m}+\sum_{\mathbf{k}, m} \tilde{\varepsilon}_{\mathbf{k}} c_{\mathbf{k} m}^{\dagger} c_{\mathbf{k} m}+\tilde{E}
$$

where

$$
\begin{aligned}
& \tilde{\varepsilon}_{f}=\varepsilon_{f}-\frac{\nu_{f}}{N} \sum_{\mathbf{k}} \frac{\left|V_{k}\right|^{2}\left\langle n_{\mathbf{k} m}^{c}\right\rangle}{\tilde{\varepsilon}_{\mathbf{k}}-\tilde{\varepsilon}_{f}}, \\
& \tilde{\varepsilon}_{\mathbf{k}}=\varepsilon_{\mathbf{k}}+\frac{\left|V_{k}\right|^{2}\left\langle\mathcal{P}_{0}(i)\right\rangle}{\tilde{\varepsilon}_{\mathbf{k}}-\tilde{\varepsilon}_{f}}, \\
& \tilde{E}=N\left(1-\left\langle\mathcal{P}_{0}(i)\right\rangle\right)\left(\varepsilon_{f}-\tilde{\varepsilon}_{f}\right) .
\end{aligned}
$$

As before, the final model $\mathcal{H}_{(\lambda \rightarrow 0)}$ consists of noninteracting $f$ and conduction electrons with renormalized one-particle energies $\tilde{\varepsilon}_{f}$ and $\tilde{\varepsilon}_{\mathbf{k}}$. The expectation values $\left\langle\mathcal{P}_{0}(i)\right\rangle=1-\left\langle n_{i}^{f}\right\rangle$ and $\left\langle n_{\mathbf{k} m}^{c}\right\rangle$ in Eqs. (58) and (60) can again be evaluated from the free energy (for details see Appendix B).

Let us solve Eq. (59) for $\tilde{\varepsilon}_{\mathbf{k}}$. We find two solutions

$$
\begin{aligned}
\tilde{\varepsilon}_{\mathbf{k}(1,2)} & =\frac{1}{2}\left\{\varepsilon_{\mathbf{k}}+\tilde{\varepsilon}_{f} \mp \sqrt{\left(\varepsilon_{\mathbf{k}}-\tilde{\varepsilon}_{f}\right)^{2}+4\left|V_{\mathbf{k}}\right|^{2}\left\langle\mathcal{P}_{0}(i)\right\rangle}\right\} \\
& =\frac{1}{2}\left\{\varepsilon_{\mathbf{k}}+\tilde{\varepsilon}_{f} \mp W_{\mathbf{k}}\right\}
\end{aligned}
$$

which agree with the well-known two heavy quasiparticle bands known from the slave-boson formalism [12]. In the following let us assume that the renormalized $f$ level $\tilde{\varepsilon}_{f}$ lies slightly above the chemical potential $\mu$ whereas the lower quasiparticle band $\tilde{\varepsilon}_{\mathbf{k}(1)}$ is intersected by $\mu$. As is easily seen our result is somewhat different from that obtained in slave-boson mean-field theory. The renormalized one-particle excitation $\tilde{\varepsilon}_{\mathbf{k}}$ in Eq. (61) agrees with the lower heavy quasiparticle band of the slave-boson theory $\tilde{\varepsilon}_{\mathbf{k}}=\tilde{\varepsilon}_{\mathbf{k}(1)}$. However, the renormalized $f$ electron excitation $\tilde{\varepsilon}_{f}$ is different from the upper heavy quasiparticle band. Instead we have found a dispersion less excitation which is located slightly above the Fermi level (see appendix B). Note that for temperature $T=0$ both approaches lead to same result for thermodynamic quantities since at zero temperature only the lower quasiparticle part is filled up to the Fermi level. However, static properties for $T \neq 0$ as well as dynamic correlation function turn out to be different within the two approaches.

\section{Transformed operators and electron Green's function}

To evaluate dynamic and different static quantities we have again to apply the transformation on operator quan- tities inside the expectation values. For the one-particle Green's functions we need

$$
c_{\mathbf{k} m}^{\dagger}(\lambda)=e^{X_{\lambda}} c_{\mathbf{k} m}^{\dagger} e^{-X_{\lambda}}
$$

and also $f_{i m}^{\dagger}(\lambda)$. In first order in the hybridization $V_{\mathbf{k}}$ one finds for the conduction electron creation operator

$$
\begin{aligned}
c_{\mathbf{k} m}^{\dagger}(\lambda) & \approx c_{\mathbf{k} m}^{\dagger}+\left[\frac{1}{L_{0}}\left(\mathbf{Q}_{\lambda} \mathcal{H}_{1}\right), c_{\mathbf{k} m}^{\dagger}\right] \\
& =c_{\mathbf{k} m}^{\dagger}+\frac{V_{\mathbf{k}}}{\varepsilon_{f}-\varepsilon_{\mathbf{k}}} \hat{f}_{\mathbf{k} m}^{\dagger}
\end{aligned}
$$

where $\hat{f}_{\mathbf{k} m}^{\dagger}$ is the Fourier transform of the $\hat{f}_{i m}^{\dagger}$. This result suggests to make the following ansatz

$$
c_{\mathbf{k} m}^{\dagger}(\lambda)=u_{\mathbf{k}, \lambda} c_{\mathbf{k} m}^{\dagger}+v_{\mathbf{k}, \lambda} \hat{f}_{\mathbf{k} m}^{\dagger}
$$

which should be suited to obtain a good approximation for $c_{\mathbf{k} m}^{\dagger}(\lambda)$. Because $c_{\mathbf{k} m}^{\dagger}(\lambda), c_{\mathbf{k} m}(\lambda)$ have to be fulfill the Fermi anticummutator relations, one concludes that

$$
1=\left|u_{\mathbf{k}, \lambda}\right|^{2}+\left|v_{\mathbf{k}, \lambda}\right|^{2}\left\langle\mathcal{P}_{0}(i)\right\rangle
$$

holds for all values of $\mathbf{k}$. Thereby, the approximation

$$
\left[\hat{f}_{\mathbf{k} m}^{\dagger}, \hat{f}_{\mathbf{k} m}\right]_{+}=\left\langle\mathcal{P}_{0}(i)\right\rangle
$$

was used. By using Eq. (64), the conduction electron occupation probability reads

$$
\begin{aligned}
\left\langle n_{\mathbf{k} m}^{c}\right\rangle & =\left\langle c_{\mathbf{k} m}^{\dagger} c_{\mathbf{k} m}\right\rangle=\left\langle c_{\mathbf{k} m}^{\dagger}(\lambda \rightarrow 0) c_{\mathbf{k} m}(\lambda \rightarrow 0)\right\rangle_{(\lambda \rightarrow 0)}^{(67)} \\
& =\left|u_{\mathbf{k},(\lambda \rightarrow 0)}\right|^{2} \frac{1}{1+e^{\beta \tilde{\varepsilon}_{\mathbf{k}}}}+\left|v_{\mathbf{k},(\lambda \rightarrow 0)}\right|^{2} \frac{1}{\nu_{f}+e^{\beta \tilde{\varepsilon}_{f}}}
\end{aligned}
$$

where $\langle\ldots\rangle_{(\lambda \rightarrow 0)}$ again denotes the expectation value formed with the renormalized Hamiltonian $\mathcal{H}_{(\lambda \rightarrow 0)}$. Note that

$$
\left\langle\hat{f}_{i m}^{\dagger} \hat{f}_{i m}\right\rangle_{(\lambda \rightarrow 0)}=\frac{1}{\nu_{f}+e^{\beta \tilde{\varepsilon}_{f}}}
$$

was used in Eq. (67) which differs from the Fermi distribution due to definition (42) for the modified creation and annihilation operators $\hat{f}_{i m}^{\dagger}$ and $\hat{f}_{i m}$.

On the other hand, the expectation value $\left\langle c_{\mathbf{k} m}^{\dagger} c_{\mathbf{k} m}\right\rangle$ can also be calculated by functional derivative from the free energy (for details see Appendix B). For zero temperature one obtains

$$
\left\langle n_{\mathbf{k} m}^{c}\right\rangle=\frac{1}{2}\left(1-\frac{\varepsilon_{\mathbf{k}}-\tilde{\varepsilon}_{f}}{W_{\mathbf{k}}}\right) \Theta\left(k_{F}-k\right)
$$

where $W_{\mathbf{k}}$ was defined by Eq. (61). By comparing Eq. (67) with Eq. (69) one finds $\left(k \leq k_{F}, \beta \rightarrow \infty\right)$ 


$$
\left|u_{\mathbf{k},(\lambda \rightarrow 0)}\right|^{2}=\frac{1}{2}\left(1-\frac{\varepsilon_{\mathbf{k}}-\tilde{\varepsilon}_{f}}{W_{\mathbf{k}}}\right)
$$

and

$$
\begin{aligned}
& \left|v_{\mathbf{k},(\lambda \rightarrow 0)}\right|^{2}=\frac{1}{2\left\langle\mathcal{P}_{0}(i)\right\rangle}\left(1+\frac{\varepsilon_{\mathbf{k}}-\tilde{\varepsilon}_{f}}{W_{\mathbf{k}}}\right) \\
& G_{\mathbf{k} m}(\omega)=\left\langle\left[\hat{f}_{\mathbf{k} m}, \frac{1}{\mathbf{L}-(\omega+i \eta)} \hat{f}_{\mathbf{k} m}^{\dagger}\right]_{+}\right\rangle=\left\langle\left[\hat{f}_{\mathbf{k} m}(\lambda \rightarrow 0), \frac{1}{\mathbf{L}_{(\lambda \rightarrow 0)}-(\omega+i \eta)} \hat{f}_{\mathbf{k} m}^{\dagger}(\lambda \rightarrow 0)\right]_{+}\right\rangle_{(\lambda \rightarrow 0)} \\
& \operatorname{Im} G_{\mathbf{k} m}(\omega)=\left\langle\mathcal{P}_{0}(i)\right\rangle\left\{\left\langle\mathcal{P}_{0}(i)\right\rangle\left|v_{\mathbf{k},(\lambda \rightarrow 0)}\right|^{2} \delta\left(\omega-\tilde{\varepsilon}_{\mathbf{k}}\right)\right. \\
& \left.+\left|u_{\mathbf{k},(\lambda \rightarrow 0)}\right|^{2} \delta\left(\omega-\tilde{\varepsilon}_{f}\right)\right\}
\end{aligned}
$$

where again $k \leq k_{F}$ has to be fulfilled. As is seen from Eqs. (70) and (71) for small $k$ values the peak at $\tilde{\varepsilon}_{f}$ dominates the imaginary part of the $f$-electron Green's function. The pole at $\tilde{\varepsilon}_{\mathbf{k}}$ becomes important for $k$ values near the Fermi momentum $k_{F}$.

\section{Discussion of the results}

Let us compare our results for the periodic Anderson model with those obtained from the slave-boson mean field theory. For simplicity we will restrict ourselves to the $T=0$ case. As already mentioned above, the renormalized energy $\tilde{\varepsilon}_{\mathbf{k}}$ corresponds to the lower quasiparticle bands from the slave-boson mean field treatment. Furthermore, the renormalized Hamiltonian $\mathcal{H}_{(\lambda \rightarrow 0)}$ leads to same $f$ state occupation as the slave-boson theory (for details see Appendix B). Moreover, the typical Kondo peak at the Fermi level is found in the imaginary part of the $f$ electron Green's function (74). Therefore, the renormalization approach for the periodic Anderson model leads at $T=0$ to the same results as the slave-boson mean field theory. On the other hand, some differences occur. The most important one is the additional quasi-particle band at the renormalized $f$ level $\tilde{\varepsilon}_{f}$ which displaces the upper heavy quasiparticle band from the slave-boson mean-field treatment. Note however that in the present approach we have neglected all processes which include different $f$ sites. A detailed discussion will be given in a forthcoming paper [13].

\section{CONCLUSION}

In summary, in this paper we have presented a renormalization approach to many-particle Hamiltoni- where condition (65) was used. For the transformed $f$ electron creation operator one finds

$$
\hat{f}_{\mathbf{k} m}^{\dagger}(\lambda)=-\left\langle\mathcal{P}_{0}(i)\right\rangle v_{\mathbf{k}, \lambda} c_{\mathbf{k} m}^{\dagger}+u_{\mathbf{k}, \lambda} \hat{f}_{\mathbf{k} m}^{\dagger}
$$

where once again Eq. (66) was used. We are now able to determine the $f$ electron Green's function

ans. First, the elimination of high energy transition operators larger than an energy cutoff $\lambda$ leads to a transformed Hamiltonian $\mathcal{H}_{\lambda}$ which is band diagonal with respect to the eigenbasis of its unperturbed part. The approach, which is based on perturbation theory, can be extended to establish a renormalization approach by continuously shifting the energy cutoff $\lambda$ to lower and lower values. In this way flow equations for the Hamiltonian are obtained. The present approach has some similarities with recently introduced renormalization method by Wegner [1] and by Glatzek and Wilson [3,4]. However, there are also some substantial differences: The present method starts from unitary transformations which are directly applied to the Hamiltonian. In contrast, the previous approach is formulated in matrix notation. It starts from flow equations in differential form for the Hamiltonian as well as for the generator of the unitary transformation. The present flow equations for the Hamiltonian can be formulated independent from a concrete representation. For demonstration the method was first applied to the exactly solvable Fano-Anderson model and to the Anderson-lattice model. For the first model the exact results were found. For the second model some approximations had to be done. For this case the well-known quasiparticle behavior of heavy fermions was rederived. In particular, it was shown that there is an almost complete agreement with the results from the slaved-boson mean-field theory [12].

\section{ACKNOWLEDGMENTS}

We would like to acknowledge fruitful discussions with F. Anders, W. Brenig, P. Fulde, N. Grewe, C. Kühnert, K. Meyer, A. Muramatsu, and S. Sykora. This work was supported by DFG through the research program SFB 463.

\section{APPENDIX A: FLOW EQUATION IN MATRIX NOTATION}

Let us start from the eigenvalue problem of the unperturbed Hamiltonian $\mathcal{H}_{0, \lambda}$ after all transitions with ener- 
gies larger than $\lambda$ have been integrated out

$$
\mathcal{H}_{0, \lambda}|n(\lambda)\rangle=E_{n, \lambda}^{0}|n(\lambda)\rangle
$$

The eigenvectors of $\mathcal{H}_{0, \lambda}$ may depend on $\lambda$. We consider two degenerate eigenstates $|n(\lambda)\rangle,|\tilde{n}(\lambda)\rangle$ belonging to the same energy $E_{n, \lambda}^{0}=E_{\tilde{n}, \lambda}^{0}$. Let us assume that the eigenstates have been orthogonalized, i.e., $\langle n(\lambda) \mid \tilde{n}(\lambda)\rangle=$ $\delta_{n, \tilde{n}}$. We multiply Eq. (16) from the left with $\langle n(\lambda)|$ and from the right with $|\tilde{n}(\lambda)\rangle$. For the renormalization of $\mathcal{H}_{0, \lambda}$ due to the elimination of excitations within the energy shell between $\lambda-\Delta \lambda$ and $\lambda$ one finds

$$
\begin{aligned}
& \left\langle n(\lambda)\left|\left(\mathcal{H}_{0,(\lambda-\Delta \lambda)}-\mathcal{H}_{0, \lambda}\right)\right| \tilde{n}(\lambda)\right\rangle= \\
& =-\sum_{\substack{m \\
\lambda-\Delta \lambda<\left|E_{n, \lambda}^{0}-E_{m, \lambda}^{0}\right| \leq \lambda}} \frac{\left\langle n(\lambda)\left|\mathcal{H}_{1, \lambda}\right| m(\lambda)\right\rangle\left\langle m(\lambda)\left|\mathcal{H}_{1, \lambda}\right| \tilde{n}(\lambda)\right\rangle}{E_{m, \lambda}^{0}-E_{n, \lambda}^{0}} .
\end{aligned}
$$

Note that the energy constraint $\lambda-\Delta \lambda<\left|E_{n, \lambda}^{0}-E_{m, \lambda}^{0}\right| \leq$ $\lambda$ may also be written as difference of two $\Theta$ functions

$$
\begin{gathered}
\sum_{\substack{\lambda-\Delta \lambda<\left|E_{n, \lambda}^{0}-E_{m, \lambda}^{0}\right| \leq \lambda \\
=}} \cdots \quad= \\
\quad \begin{array}{l}
\sum \cdots\left[\left|E_{n, \lambda}^{0}-E_{m, \lambda}^{0}\right|-(\lambda-\Delta \lambda)\right] \\
\left.-\Theta\left(\left|E_{n, \lambda}^{0}-E_{m, \lambda}^{0}\right|-\lambda\right)\right\}
\end{array}
\end{gathered}
$$

so that on the rhs of Eq. (A2) the limit $\Delta \lambda \rightarrow 0$ can be easily performed. For the lhs it is easy to verify that the limit $\Delta \lambda \rightarrow 0$ leads to the derivative of $E_{n, \lambda}^{0}$ with respect to $\lambda$. Here, the orthogonality of the vectors $|n(\lambda)\rangle,|\tilde{n}(\lambda)\rangle$ has to be used. Thus, one finds

$$
\begin{aligned}
-\frac{d E_{n, \lambda}^{0}}{d \lambda}= & -\sum_{m} \frac{\left\langle n(\lambda)\left|\mathcal{H}_{1, \lambda}\right| m(\lambda)\right\rangle\left\langle m(\lambda)\left|\mathcal{H}_{1, \lambda}\right| \tilde{n}(\lambda)\right\rangle}{E_{m, \lambda}^{0}-E_{n, \lambda}^{0}} \\
& \times \frac{d \Theta\left(\left|E_{n, \lambda}^{0}-E_{m, \lambda}^{0}\right|-\lambda\right)}{d \lambda}
\end{aligned}
$$

In Eq. (A4) the $\lambda$ derivative of the $\Theta$ function acts on the explicit term linear in $\lambda$ and on the $\lambda$-dependent energies $E_{n, \lambda}^{0}$ and $E_{m, \lambda}^{0}$. It is obvious that the latter dependence leads to higher order contributions in $\mathcal{H}_{1, \lambda}$. Therefore, by restricting one-self to the lowest non vanishing order in $\mathcal{H}_{1, \lambda}$ one finds

$$
\begin{aligned}
\frac{d E_{n, \lambda}^{0}}{d \lambda}= & -\sum_{m}\left\langle n(\lambda)\left|\mathcal{H}_{1, \lambda}\right| m(\lambda)\right\rangle\left\langle m(\lambda)\left|\mathcal{H}_{1, \lambda}\right| \tilde{n}(\lambda)\right\rangle \\
& \times \frac{1}{E_{m, \lambda}^{0}-E_{n, \lambda}^{0}} \delta\left(\left|E_{n, \lambda}^{0}-E_{m, \lambda}^{0}\right|-\lambda\right) .
\end{aligned}
$$

Note that flow equations for the $\lambda$ dependent matrix elements of the interaction $\mathcal{H}_{1, \lambda}$ can be derived in a similar way.

\section{APPENDIX B: CALCULATION OF THE OCCUPATION PROBABILITIES}

In this appendix we derive formal expressions for the particle number expectation values for the periodic Anderson model. Thereby, we restrict ourselves to zero temperature. Let us assume that the renormalized $f$ level $\tilde{\varepsilon}_{f}$ lies slightly above the chemical potential $\mu$ whereas the lower quasiparticle band $\tilde{\varepsilon}_{\mathbf{k}(1)}$ is intersected by $\mu$. Thus for $T=0$, according to Eqs. (57) and (60) the groundstate energy $E_{g}=F(T \rightarrow 0)$ is given by

$$
\begin{aligned}
E_{g} & =\tilde{E}+\nu_{f} \sum_{k \leq k_{F}} \tilde{\varepsilon}_{\mathbf{k}} \\
& =N\left(1-\left\langle\mathcal{P}_{0}(i)\right\rangle\right)\left(\varepsilon_{f}-\tilde{\varepsilon}_{f}\right)+\nu_{f} \sum_{k \leq k_{F}} \tilde{\varepsilon}_{\mathbf{k}}
\end{aligned}
$$

where $k_{F}$ denotes the Fermi momentum and $\tilde{\varepsilon}_{\mathbf{k}(1)}=\tilde{\varepsilon}_{\mathbf{k}}$. The ground state energy can be used to calculate the zero-temperature occupation probabilities. The functional derivative of Eq. (57) leads to

$$
\begin{aligned}
\left\langle n_{i}^{f}\right\rangle & =\frac{1}{N} \frac{\partial E_{g}}{\partial \varepsilon_{f}}=\frac{1}{N} \frac{\partial \tilde{E}}{\partial \varepsilon_{f}}+\frac{\nu_{f}}{N} \sum_{k \leq k_{F}} \frac{\partial \tilde{\varepsilon}_{\mathbf{k}}}{\partial \varepsilon_{f}} \text { and } \\
\left\langle n_{\mathbf{k} m}^{c}\right\rangle & =\frac{1}{\nu_{f}} \frac{\partial E_{g}}{\partial \varepsilon_{\mathbf{k}}}=\frac{1}{\nu_{f}} \frac{\partial \tilde{E}}{\partial \varepsilon_{\mathbf{k}}}+\sum_{k^{\prime} \leq k_{F}} \frac{\partial \tilde{\varepsilon}_{\mathbf{k}^{\prime}}}{\partial \varepsilon_{\mathbf{k}}}
\end{aligned}
$$

where due to Eq. (60)

$$
\frac{\partial \tilde{E}}{\partial \varepsilon_{f}}=N\left\langle n_{i}^{f}\right\rangle\left(1-\frac{\partial \tilde{\varepsilon}_{f}}{\partial \varepsilon_{f}}\right)+N\left(\varepsilon_{f}-\tilde{\varepsilon}_{f}\right) \frac{\partial\left\langle n_{i}^{f}\right\rangle}{\partial \varepsilon_{f}} .
$$

Furthermore, by using Eq. (61) one obtains

$$
\frac{\partial \tilde{\varepsilon}_{\mathbf{k}}}{\partial \varepsilon_{f}}=\frac{1}{2}\left(1+\frac{\varepsilon_{\mathbf{k}}-\tilde{\varepsilon}_{f}}{W_{\mathbf{k}}}\right) \frac{\partial \tilde{\varepsilon}_{f}}{\partial \varepsilon_{f}}+\frac{\left|V_{\mathbf{k}}\right|^{2}}{W_{\mathbf{k}}} \frac{\partial\left\langle n_{i}^{f}\right\rangle}{\partial \varepsilon_{f}} .
$$

By inserting Eqs. (B4) and (B5) into Eq. (B2) one finds

$$
\begin{aligned}
0= & \frac{\partial \tilde{\varepsilon}_{f}}{\partial \varepsilon_{f}}\left[-\left\langle n_{i}^{f}\right\rangle+\frac{1}{2} \frac{\nu_{f}}{N} \sum_{k \leq k_{F}}\left(1+\frac{\varepsilon_{\mathbf{k}}-\tilde{\varepsilon}_{f}}{W_{\mathbf{k}}}\right)\right] \\
& +\frac{\partial\left\langle n_{i}^{f}\right\rangle}{\partial \varepsilon_{f}}\left[\varepsilon_{f}-\tilde{\varepsilon}_{f}+\frac{\nu_{f}}{N} \sum_{k \leq k_{F}} \frac{\left|V_{\mathbf{k}}\right|^{2}}{W_{\mathbf{k}}}\right] .
\end{aligned}
$$

We can conclude

$$
\left\langle n_{i}^{f}\right\rangle=\frac{1}{2} \frac{\nu_{f}}{N} \sum_{k \leq k_{F}}\left(1+\frac{\varepsilon_{\mathbf{k}}-\tilde{\varepsilon}_{f}}{W_{\mathbf{k}}}\right)
$$

and

$$
\tilde{\varepsilon}_{f}-\varepsilon_{f}=\frac{\nu_{f}}{N} \sum_{k \leq k_{F}} \frac{\left|V_{\mathbf{k}}\right|^{2}}{W_{\mathbf{k}}}
$$


Thus the $f$ state occupation of the slave boson meanfield theory is reobtained [12]. $\left\langle n_{\mathbf{k} m}^{c}\right\rangle$ can be found in the same way

$$
\begin{aligned}
\left\langle n_{\mathbf{k} m}^{c}\right\rangle= & \frac{1}{2}\left(1-\frac{\varepsilon_{\mathbf{k}}-\tilde{\varepsilon}_{f}}{W_{\mathbf{k}}}\right) \Theta\left(k_{F}-k\right) \\
& +\frac{\partial \tilde{\varepsilon}_{f}}{\partial \varepsilon_{\mathbf{k}}}\left[-\frac{N}{\nu_{f}}\left\langle n_{i}^{f}\right\rangle+\frac{1}{2} \sum_{k^{\prime} \leq k_{F}}\left(1+\frac{\varepsilon_{\mathbf{k}^{\prime}}-\tilde{\varepsilon}_{f}}{W_{\mathbf{k}^{\prime}}}\right)\right] \\
& +\frac{\partial\left\langle n_{i}^{f}\right\rangle}{\partial \varepsilon_{\mathbf{k}}}\left[\frac{N}{\nu_{f}}\left(\varepsilon_{f}-\tilde{\varepsilon}_{f}\right)+\sum_{k^{\prime} \leq k_{F}} \frac{\left|V_{\mathbf{k}^{\prime}}\right|^{2}}{W_{\mathbf{k}^{\prime}}}\right]
\end{aligned}
$$

By using Eqs. (B7) and (B8) one finally obtains

$$
\left\langle n_{\mathbf{k} m}^{c}\right\rangle=\frac{1}{2}\left(1-\frac{\varepsilon_{\mathbf{k}}-\tilde{\varepsilon}_{f}}{W_{\mathbf{k}}}\right) \Theta\left(k_{F}-k\right) .
$$

[1] F. Wegner, Ann. Phys. (Leipzig) 3, 77 (1994).

[2] A. Mielke, Euro. Phys. J. B 5, 605 (1998).

[3] S.D. Głazek and K.G. Wilson, Phys. Rev. D 48, 5863 (1993).

[4] S.D. Głazek and K.G. Wilson, Phys. Rev. D 49, 4214 (1994).

[5] I. Grote, E. Körding, and F. Wegner, J. Low Temp. Phys. 126, 1385 (2002).

[6] P.W. Anderson, Phys. Rev. 124, 41 (1961).

[7] U. Fano, Phys. Rev. 124, 1866 (1961).

[8] See, for example, M. Takahashi, J. Phys. C: Solid State Phys. 10, 1289 (1977).

[9] S. Sykora, A. Hübsch, and K. W. Becker, to be published.

[10] C. Knetter and G. S. Uhrig, Eur. Phys. J. B 13, 209 (2000).

[11] A.C. Hewson, The Kondo Problem to Heavy Fermions, Cambridge Studies in Magnetism, Cambridge University Press, Cambridge, 1993.

[12] See, for example, P. Fulde, J. Keller, and G. Zwicknagl: In Solid State Physics, Vol. 41, ed. by H. Ehrenreich and D. Turnbull (Academic, San Diego 1988), p. 1.

[13] T. Sommer, K. Meyer, A. Hübsch, and K. W. Becker, to be published. 